\title{
Domoic Acid Lesions in Nucleus of the Solitary Tract: Time-Dependent Recovery of Hypoxic Ventilatory Response and Peripheral Afferent Axonal Plasticity
}

\author{
Zixi (Jack) Cheng, ${ }^{1}$ Shang Z. Guo, ${ }^{1}$ Andrew J. Lipton, ${ }^{1}$ and David Gozal ${ }^{1,2}$ \\ ${ }^{1}$ Kosair Children's Hospital Research Institute, Department of Pediatrics, and ${ }^{2}$ Department of Pharmacology and \\ Toxicology, University of Louisville School of Medicine, Louisville, Kentucky 40202
}

The nucleus of the solitary tract (NTS) plays a pivotal role in the ventilatory response to hypoxia (HVR). However, the effects of excitotoxic lesions and the potential for functional recovery and plasticity remain unknown. Domoic acid (DA) or vehicle were bilaterally injected within the NTS of adult male Sprague Dawley rats. HVR $\left(10 \% \mathrm{O}_{2}\right)$ and anatomical changes were assessed at 5-90 d after surgery. DA induced dose-dependent HVR attenuations ( $\sim 70 \%$ at peak effect) that exhibited saturation at concentrations of $0.75-1.0 \mathrm{~mm}$. However, although sodium cyanide-induced ventilatory responses were virtually abolished, DA did not modify baroreceptor gain. Consistent with ventilatory reductions, NTS neurons showed a significant degeneration $3 \mathrm{~d}$ after DA injection. In addition, the projection fields and the density of vagal afferent terminals to the NTS, and the motor neurons in the dorsal motor nucleus of the vagus were substantially reduced at $15 \mathrm{~d}$. At $30 \mathrm{~d}$, no functional or neural recovery were apparent. However, at day 60 , the reduction in HVR was only $\sim 40 \%$ of control, and at $90 \mathrm{~d}$, HVR returned to control levels, paralleling regeneration of vagal afferent terminals within the NTS. The regeneration was particularly prominent in the commissural and dorsomedial subnuclei in the absence of cellular recovery. Thus, the integrity of the NTS is critical for HVR, spontaneous HVR recovery occurs after excitotoxic lesions in the NTS, and vagal-glossopharyngeal terminal sprouting in the NTS may underlie the anatomical substrate for such spontaneous functional recovery. The adult brainstem/ NTS has self-repairing capabilities and will compensate for functional losses after structural damage by rewiring of its neural circuitry.

Key words: hypoxic ventilatory response; brainstem; glutamate; excitotoxicity; baroreceptor; functional plasticity
Disruption of normal cardiorespiratory regulatory mechanisms by any of a multiplicity of disorders may lead to substantial mortality and morbidity (Kales et al., 1985; Gozal, 1998; De Caro et al., 2000; Nagata et al., 2000; Derrick et al., 2001; Gozal et al., 2001). Therefore, the study of the functional consequences of targeted brainstem lesions and their potential for functional and anatomical recovery emerges as a particularly important area of research.

Whether the adult CNS may recover after damage is a debatable issue. It is generally accepted that CNS lesions lead to functional deficits that fail to improve over time (Goldberg and Barres, 2000a,b). More recently however, it has been demonstrated that spontaneous functional recovery does occur in the CNS (Björklund and Lindvall, 2000; Chan et al., 2000; Magavi et al., 2000; Weidner et al., 2001). For example, Weidner et al. (2001) demonstrated that spontaneous corticospinal axonal sprouting onto the motor neurons in the cervical spinal cord had occurred after transecting dorsal corticospinal motor pathways, and this axonal plasticity was in parallel with the recovery of skilled forelimb movement. In neocortex, Magavi et al. (2000)

\footnotetext{
Received Sept. 18, 2001; revised Jan. 10, 2002; accepted Feb. 4, 2002.

Z.J.C. is supported by American Heart Association Grant 9930173N, and D.G. receives support from National Institutes of Health Grants HL-63912, HL-65270, and HL-66358, American Heart Association Grant 0050442N, and The Commonwealth of Kentucky Research Challenge Trust Fund.

Correspondence should be addressed to Dr. David Gozal, Kosair Children's Hospital Research Institute, Department of Pediatrics, 570 South Preston Street, Suite 321, University of Louisville School of Medicine, Louisville, KY 40202. E-mail: david.gozal@louisville.edu.

Copyright (C) 2002 Society for Neuroscience $\quad 0270-6474 / 02 / 223215-12 \$ 15.00 / 0$
}

destroyed a subset of pyramidal neurons that project from the neocortex to the thalamus. Two weeks later, these investigators observed newly formed neurons in the damaged neocortex and some of these neurons extended processes to the original target sites in the thalamus, suggesting that they had been integrated in the neural circuitry. In the nucleus of the solitary tract (NTS), Chan et al. (2000) studied restoration of c-fos expression induced by phenylephrine infusion in chronically carotid sinus nerve denervated (CSND) rats. At $30 \mathrm{~d}$ after CSND, normal pattern of Fos induction in response to phenylephrine infusion was observed, suggesting that peripheral nerve synaptic reorganization had occurred in the NTS after partial baroreceptor nerve denervation.

The functional roles of the NTS in integrating sensory inputs and initiating homeostatic responses by way of influences on presympathetic neurons have been studied extensively (for review, see Guyenet, 2000). However, the importance of NTS integrity in respiratory reflexes and its potential in functional recovery over time have not been specifically addressed. Indeed, Housley and Sinclair (1988) injected the neurotoxin kainic acid into the caudal NTS and found significant reductions in ventilatory response to hypoxia (HVR). However, these investigators did not explore whether functional recovery occurs after excitotoxic NTS lesions.

In the present study, we lesioned the NTS with domoic acid (DA), a potent neurotoxin for cells harboring glutamate receptors, and tested two major hypotheses: namely that the integrity of the NTS is crucially important for the HVR and peripheral chemoreceptor-mediated ventilatory responses, and that the le- 
sioned NTS may have a certain degree of self-repair capacity and plasticity, which if allowed to proceed will manifest as functional and anatomical recovery.

\section{MATERIALS AND METHODS}

The experimental protocols were approved by the Institutional Animal Care and Use Committee of the University of Louisville. Young adult male Sprague Dawley rats (250-300 gm) were obtained from a commercial breeder (Charles River). All efforts were made to minimize the animal suffering and to reduce the number of animals used.

Injections of DA in the NTS. Animals were anesthetized with pentobarbital sodium $(60 \mathrm{mg} / \mathrm{kg}$, i.p. $)$, treated with atropine $(1 \mathrm{mg} / \mathrm{kg}$, s.c. $)$, intubated, and placed in a stereotaxic instrument with a head holder adapted to permit downward and forward flexion of the neck. Under mechanical ventilation via the endotracheal tube, a dorsal incision was performed over the dorsal neck muscles, which were then retracted to expose the atlanto-occipital membrane. The membrane was opened to expose the cisterna magna and the dorsal medulla, and the occipital bone was trimmed with the bit of a dental drill until the caudal cerebellum became visible. The obex was used as reference for stereotactic coordinates. A glass micropipette [inner diameter (ID) $10-20 \mu \mathrm{m}$ ] filled with domoic acid and connected to a picospritzer was then advanced with a micromanipulator to the NTS under direct visualization. DA was pressure-injected bilaterally in small aliquots $(\cong 5-12.5 \mathrm{nl}$ each) at 16 different sites ( 8 left and $8 \mathrm{right} ;-800$ to $+800 \mu \mathrm{m}$; total volume $\cong 80-200 \mathrm{nl}$ ), separated $\sim 375 \mu \mathrm{m}$ longitudinally along the NTS. After injections, the atlanto-occipital membrane was microsutured to prevent any leak of cerebrospinal fluid, the surgical wound was closed, and cardiorespiratory parameters were monitored for $2-4 \mathrm{hr}$ or until the animal exhibited stable spontaneous respiration and heart rate. Animals were then returned to their cages, injected intraperitoneally with $2.5 \mathrm{cc}$ of normal saline bilaterally, and provided with ad libitum access to water and rat chow.

Dose-dependent effect of DA on HVR. To test the hypothesis that DA would be associated with a dose-dependent effect on HVR, animals were randomly assigned to receive PBS, which served as control, or $0.10,0.25$, $0.50,0.75,1.00$, and $1.25 \mathrm{~mm}$ DA.

HVR was tested $1 \mathrm{~d}$ before surgery and 5 and $15 \mathrm{~d}$ after DA microinjections into the NTS. For each dose, at least six rats were included. These experiments permitted delineation of the optimal dose of DA for subsequent experiments.

Effect of DA on hypoxic and hypercapnic ventilatory responses. Respiratory measures were continuously acquired in the freely behaving, unrestrained animal placed in a previously calibrated 21 barometric chamber, using the methods described by Bartlett and Tenney (1970) and Pappenheimer (1977). To minimize the long-term effect of signal drift caused by temperature and pressure changes outside the chamber, a reference chamber of equal size in which temperature was measured using a $\mathrm{T}$ type thermocouple was used. In addition, as recommended previously by Epstein and colleagues (1980), a correction factor was incorporated into the software routine to account for inspiratory and expiratory barometric asymmetries. Environmental temperature was maintained within 24$26^{\circ} \mathrm{C}$. A calibration volume of $0.5 \mathrm{ml}$ of air was repeatedly introduced into the chamber before and after completion of recordings. At least 30 min before the start of each protocol, animals were allowed to acclimate to the chamber, in which humidified air ( $90 \%$ relative humidity) warmed at $30^{\circ} \mathrm{C}$ was passed through at a rate of $21 / \mathrm{min}$, using a precision flow pump-reservoir system. Pressure changes in the chamber caused by the inspiratory and expiratory temperature changes (Drorbaugh and Fenn, 1955) were measured using a high-gain differential pressure transducer (Validyne, Model MP45-1). Analog signals were continuously digitized and analyzed on-line by a microcomputer software program (Buxco Electronics, Troy, NY). A rejection algorithm was included in the breathby-breath analysis routine and allowed for accurate rejection of motioninduced artifacts. Minute ventilation was computed and stored for subsequent off-line analysis. Recordings were performed in room air and during a $10 \mathrm{~min}$ exposure to $10 \% \mathrm{O}_{2}$ in $\mathrm{N}_{2}$ or a $15 \mathrm{~min}$ exposure to $5 \%$ $\mathrm{CO}_{2}$ balanced in room air using preset gas mixtures.

Effect of DA on baroreceptor and chemoreceptor function. To examine whether DA microinjections into the NTS are associated with baroreceptor and peripheral chemoreceptor dysfunction, DA was injected at 1 $\mathrm{mm}$ concentration in 32 additional rats. On day 9 after DA microinjection, rats were anesthetized, and indwelling catheters [PE50, $0.56 \mathrm{~mm}$ $\mathrm{ID}, 0.88 \mathrm{~mm}$ outer diameter (OD)] were introduced into the femoral artery and vein and advanced into the abdominal aorta and inferior vena cava, respectively. Catheters were secured in the groin area with sutures, tunneled under the skin into the dorsal neck region, flushed with a heparin-containing solution (1000 $\mathrm{U} / \mathrm{ml}$ saline), sealed with heat, and stored in a plastic cap sutured to the skin.

Recordings were conducted while the animals were still under anesthesia as evidenced by the absence of eye reflex and paw removal to a noxious stimulus, and identical experiments were also conducted in the freely behaving conscious animals $\sim 48 \mathrm{hr}$ later. We have shown previously that full resumption of grooming, ingestive, and other behaviors occurs after this recovery period (Gozal et al., 1996a). In six DA-treated rats and in six control animals, the HVR was measured as above, and arterial blood gases were measured from a blood sample drawn during room air conditions and at the 10th min of hypoxic exposure. After withdrawal of $75-100 \mu \mathrm{l}$ of blood in the dead space of the catheter, another $150 \mu \mathrm{l}$ was sampled for immediate analysis of $\mathrm{P}_{\mathrm{O}_{2}}, \mathrm{P}_{\mathrm{CO}_{2}}$, and $\mathrm{pH}$ with a blood gas analyzer (Radiometer, ABL510, Copenhagen, Denmark).

For assessment of peripheral chemoreceptor function, sodium cyanide $(\mathrm{NaCN})$ was rapidly injected in the venous catheter $(<1 \mathrm{sec})$ at $40-80$ $\mu \mathrm{g} / \mathrm{kg}$ in $0.5 \mathrm{ml}$ aliquots $(n=6$ per group for both DA and control groups), as described previously (Gozal et al., 1996b). Ventilatory measures were acquired as detailed above. For initial determinations of baroreceptor function, phenylephrine ( 0 or $40 \mu \mathrm{g} / \mathrm{kg}$ in $0.5 \mathrm{ml}$ aliquots) was rapidly injected ( $n=6$ per group for both DA and control groups), and the magnitude of cardiac deceleration as a function of mean arterial blood pressure increase was determined from the mean of three separate trials in each animal. In addition, in another set of 12 anesthetized rats (6 DA- and 6 saline-treated), administration of the vasoactive drugs phenylephrine and sodium nitroprusside, for activation or deactivation of baroreceptors, was performed every $60-120 \mathrm{sec}$ (solution concentration, $100 \mu \mathrm{g} / \mathrm{ml}$; inf usion rate, $20,30,40$, or $60 \mu \mathrm{l} / \mathrm{min}$ ). The mean steady-state values during the 20 beats preceding each dose administration were considered as baseline. At least 15 min were allowed after phenylephrine infusion and $30 \mathrm{~min}$ after sodium nitroprusside infusion to allow for the hemodynamic parameters to return to baseline. In all animals, both arterial blood pressure and heart rate were measured from the arterial line connected to a calibrated pressure transducer. The analog signal was digitized and signal processed using peak-trough software routines to derive mean arterial blood pressure and heart rate on a beat-to-beat mode (Buxco Electronics).

Ventilatory and cardiovascular data analysis. Values are reported as mean $\pm \mathrm{SD}$. Baseline ventilation before each hypoxic or $\mathrm{NaCN}$ run was defined as the average of ventilatory measures during the $3 \mathrm{~min}$ period immediately preceding the challenge. For ventilatory challenges, the peak ventilation value (sustained for $1 \mathrm{~min}$ in hypoxic challenges and for five consecutive breaths for $\mathrm{NaCN}$ challenges) was considered as representative of the ventilatory response. To normalize across the various experiments, the overall peak ventilation increase was calculated using the mean baseline values preceding each challenge and was therefore expressed as percentage baseline.

Absolute values for heart rate (beats per minute) were used to evaluate the arterial baroreflex during changes in blood pressure. For each instantaneous heart rate value (HR), the corresponding mean arterial pressure (MAP) was calculated. The MAP was plotted against HR for each of the drug doses. Data points representing the spontaneous changes in MAP and corresponding reflex changes in HR were fit by a linear regression. Data points were averaged for each animal and then for the group. The slope of the regression was used as an index of baroreflex sensitivity (beats per minute per millimeters mercury, pressure). Although the data can be reliably presented by a linear regression equation, the baroreflex regulation of $\mathrm{HR}$ is generally better expressed by constructing a logistic function curve compiled from data obtained by intravenous infusion in increasing doses of phenylephrine and sodium nitroprusside (Kent et al., 1972). Therefore, the data from each rat were also fitted to a sigmoid logistic function described by the following equation: $\mathrm{HR}=\mathrm{P}_{4}+$ $\left\{\left(P_{1}\right) / 1+\exp \left[P_{2}\left(\mathrm{MAP}-P_{3}\right)\right]\right\}$, where $P_{1}$ is the range of $\mathrm{HR}, \mathrm{P}_{2}$ is the coefficient to calculate the gain as a function of pressure, $P_{3}$ is the pressure at the midrange of curve, and $\mathrm{P}_{4}$ is the minimum response of HR. The gain at any given MAP was then calculated using the equation: Gain $+P_{1} P_{2}\left\{\exp \left[P_{2}\left(\mathrm{MAP}-P_{3}\right)\right]\right\} /\left\{1+\exp \left[P_{2}\left(\mathrm{MAP}-P_{3}\right)\right]\right\} 2$.

Neuronal degeneration, functional, and anatomical recovery experiments. For neurodegeneration, anatomical, and functional recovery studies, all DA microinjections in the NTS were conducted using the $1 \mathrm{~mm}$ concentration. This dose was selected on the basis of dose-dependent experiments described above and their effect on HVR. Three survival periods 
were allowed after DA or control injections: namely $3 \mathrm{~d}(n=6$ per group), $15 \mathrm{~d}$ ( $n=6$ per group), $30 \mathrm{~d}(n=3$ per group), and $90 \mathrm{~d}(n=$ 8 per group). Animals that were allowed to survive for only $3 \mathrm{~d}$ were used to examine neuronal degeneration of NTS neurons using cupric silver staining (see below). Animals allowed to survive for up to $30 \mathrm{~d}$ underwent HVR measurements the day preceding surgery, and at 10,15, and when appropriate $30 \mathrm{~d}$ after surgery. Animals allowed to survive for $90 \mathrm{~d}$ underwent assessment of their HVR on the day preceding surgery, and at days $15,30,60$, and 90 after surgery. On completion of the last planned HVR measurement, animals were injected with the neuronal tracer tetramethylrhodamine dextran (TMR-D) and killed 1 week later. The brainstems were removed for morphological examinations of neural degeneration and regeneration in the NTS (see Anterograde tracing methods)

Finally, 10 rats were used during the initial phases of these experiments to verify the extent and location of DA injection sites in the NTS.

Cupric silver staining. Cupric silver staining was used to selectively identify degenerating neurons in the NTS region, and the procedure was adapted from previously published methods (Fix et al., 1996; Switzer, 2000). Briefly, $3 \mathrm{~d}$ after DA injections, animals were deeply anesthetized with sodium pentobarbital and perfused through the left ventricle with a special fixative solution designed for the cupric silver procedure. Brains remained in the skull and were immersed in the same fixative for another $2-4 \mathrm{~d}$ and then removed. Whole brains were cryoprotected and embedded collectively in a gelatin block (MultiBrain technology, NeuroScience Associates, Knoxville, TN), with parallel alignment of their rostral-caudal axes. Blocks were allowed to harden and were subsequently frozen with dry ice. Serial cross sections $(40 \mu \mathrm{m})$ were then taken through the entire block. Because all embedded brains had similar orientation in each gelatin block, each cut could provide a set of cross sections at the same level of the brain. This approach allowed for convenient comparisons of cupric silver-stained neurons at similar levels among brainstem sections from different animals.

For staining, sections were placed in an aqueous mixture of silver nitrate, copper nitrate, cadmium nitrate, pyridine, and ethanol and were then processed through the following sequences: acetone; silver nitratein combination with ammonium and sodium hydroxide; and a weak formaldehyde-citric acid and ethanol solution (for reduction). Sections were then bleached in potassium ferricyanide and sodium borate to removed unreduced silver. After several rinses, sections were mounted on $2 \times 3$ inch glass slides, air dried, and coverslipped. The brainstem sections were examined using a light microscope (Olympus iX50) and photographed using a digital camera (Kodak 290C).

Anterograde labeling of peripheral afferents in the NTS. The anterograde tracing technique was used to label peripheral afferent terminals in the NTS. The nodose ganglion and petrosal ganglion are very close to each other and form the vagal-petrosal complex in rats; hence, injections of a anterograde tracer into the nodose ganglion provide the most complete labeling of both vagal and glossopharyngeal afferents. TMR-D (7\%, 3000 MW; catalog number D-3308, Molecular Probes, Eugene, OR) was therefore injected into the left nodose ganglion to label unilateral peripheral afferent terminals in the NTS. It should be noted that TMR-D also serves as a retrograde tracer, such that TMR-D injected into the nodose ganglion will also label the motor neurons in the dorsal motor nucleus of the vagus (DmnX).

Animals were anesthetized with pentobarbital sodium $(60 \mathrm{mg} / \mathrm{kg}$, i.p.) and injected with atropine sulfate $(1 \mathrm{mg}$, s.c.). After each animal was unresponsive to ear pinch, its neck was shaved, a midline incision was made along the neck, and the ventral neck muscles were gently separated by blunt dissection to expose the nodose ganglion medial to the internal carotid artery, following a procedure similar to that of Cheng and colleagues (1997a). Multiple injections of TMR-D (total dose $500 \mathrm{nl}$ ) were made into the ganglion although a micropipette under continuous visual inspection using a surgical microscope and a picospritzer (54 psi; $4 \mathrm{msec} ; 20-40 \mu \mathrm{m}$ ID micropipette). After each injection, the micropipette was left in place for $1 \mathrm{~min}$ before being withdrawn to reduce dye leakage. After completion of all injections, the surgical wound was closed with sutures, and animals were returned to their cages.

After a survival period of $7 \mathrm{~d}$ to allow for tracer transport to the brainstem, each animal was anesthetized with an overdose of pentobarbital sodium $(100 \mathrm{mg} / \mathrm{kg})$. When fully unresponsive, each rat was perfused through the heart with $0.9 \%$ saline $(300 \mathrm{ml})$ and phosphate-

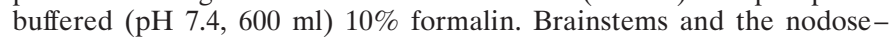
petrosal ganglion complex were removed. Each brainstem containing the entire NTS, DmnX, and nucleus of ambiguus (NA) was stored in $15 \%$ sucrose formalin overnight and sectioned transversely at $100 \mu \mathrm{m}$ using a cryostat on the second day. All tissues were then dehydrated through a graded series of ethanol rinses $(70 \%, 2 \mathrm{~min} ; 90 \%, 2 \mathrm{~min}$; and two times $100 \%, 1.5 \mathrm{~min}$ each). Finally the tissue was mounted and coverslipped in Cytoseal XYL.

Analysis of vagal brainstem terminals. The brainstem sections were coded such that the experimenter performing the microscopic analysis of the sections was unaware of the treatment received by the animals. All brainstem sections of these coded animals were initially examined with a conventional epifluorescence microscope $(20 \times$ objective). The brainstem slices at the level of $-800,-200$, and $+800 \mu \mathrm{m}$ to the obex were scanned with a laser confocal microscope (Zeiss $510 ; 25 \times$ oil lens). Stacks of optically sectioned images were scanned through dual channels (rhodamine and FITC) at the z-step of $3 \mu \mathrm{m}$. Each stack was then projected onto a single plane to give a three-dimensional (3-D) all-in-focus projection image. For each brainstem section, 25-30 stacks of optical sectioned images were collected, projected, and assembled together to make a montage and thereby present the whole terminal field of peripheral afferents in that brainstem slice. The density of TMR-D-labeled vagal terminals in the NTS was subsequently assessed from the confocal montages at the three preset planes.

Verification of DA injection sites in NTS. A double fluorescent labeling strategy was used to verify the location and estimate the extent of DA injections in the dorsal vagal complex, which included the NTS and the dorsal motor nucleus of the vagus. First, the anterograde tracer TMR-D (red) was injected into the left nodose ganglion to label the projection field of the peripheral afferent terminals, as described above. Seven days after the TMR-D injections, another fluorescent dye, 4-(4-dihexadecylamino)-styryl)- $N$-methylpyridinium iodide (DiA) (yellow-green; catalog 3883, Molecular Probes), was injected into the NTS bilaterally using the same approach as that used for DA. One hour after surgical closure of the wound, animals were perfused using the same protocol as that used for the TMR-D-injected animals. Brainstems were removed, sectioned, processed as delineated above, and examined using both conventional epifluorescence microscope and scanning obtained with the confocal microscope.

\section{RESULTS}

\section{Physiological studies}

\section{Hypoxic ventilatory response after DA injections}

Microinjections of DA within the NTS were associated with substantial albeit transient increases in arterial blood pressure and blood pressure lability during the first $24 \mathrm{hr}$ after surgery but not after $15 \mathrm{~d}$ from DA injection. These findings have been documented previously in electrolytic lesions of the NTS in cats and rats (Nathan and Reis, 1977; Rockhold and Caldwell, 1979; Buchholz and Nathan, 1984).

Compared with the response of saline-injected animals, HVRs of DA-treated animals were profoundly reduced at day $15 \mathrm{~d}$ after surgery. As shown in Figures 1 and 2, HVR reduction was dose dependent and affected both tidal volume and frequency components similarly. No further reductions in HVR occurred beyond a DA concentration of $0.75 \mathrm{~mm}$, such that $1 \mathrm{~mm}$ was selected as the optimal dose for subsequent experiments. In fact, injection of DA at a dose of $1.25 \mathrm{~mm}$ elicited uncontrolled seizures that generally led to animal death, possibly caused by very severe and complicated cardiovascular, respiratory, and gastrointestinal reflexes. Thus, DA concentrations associated with survival achieved maximal HVR reductions in the vicinity of $70 \%$ from the HVR measured before surgery. This was further confirmed by arterial blood gases that showed similar $\mathrm{pH}, \mathrm{P}_{\mathrm{CO}_{2}}$, and $\mathrm{P}_{\mathrm{O}_{2}}$ during normoxia $\left(\mathrm{pH}, 7.38 \pm 0.2\right.$ and $7.37 \pm 0.2 ; \mathrm{P}_{\mathrm{CO}_{2}}, 37.2 \pm 1.9$ and $36.9 \pm$ $1.6 \mathrm{mmHg} ; \mathrm{P}_{\mathrm{O}_{2}}, 101.7 \pm 4.6$ and $103.7 \pm 5.6 \mathrm{mmHg}$ in $\mathrm{DA}$ and vehicle-treated animals, respectively) but showed significant differences at $10 \mathrm{~min}$ hypoxia. Indeed, mean $\mathrm{pH}$ was $7.59 \pm 0.2$, $\mathrm{P}_{\mathrm{CO}_{2}}$ was $26.5 \pm 2.1 \mathrm{mmHg}$, and $\mathrm{P}_{\mathrm{O}_{2}}$ was $42.7 \pm 1.8 \mathrm{mmHg}$ in vehicle-treated rats compared with $\mathrm{pH}$ of $7.47 \pm 0.1(p<0.01)$, $\mathrm{P}_{\mathrm{CO}_{2}}$ of $33.5 \pm 1.5 \mathrm{mmHg}(p<0.01)$, and $\mathrm{P}_{\mathrm{O}_{2}}$ of $40.2 \pm 2.1$ 

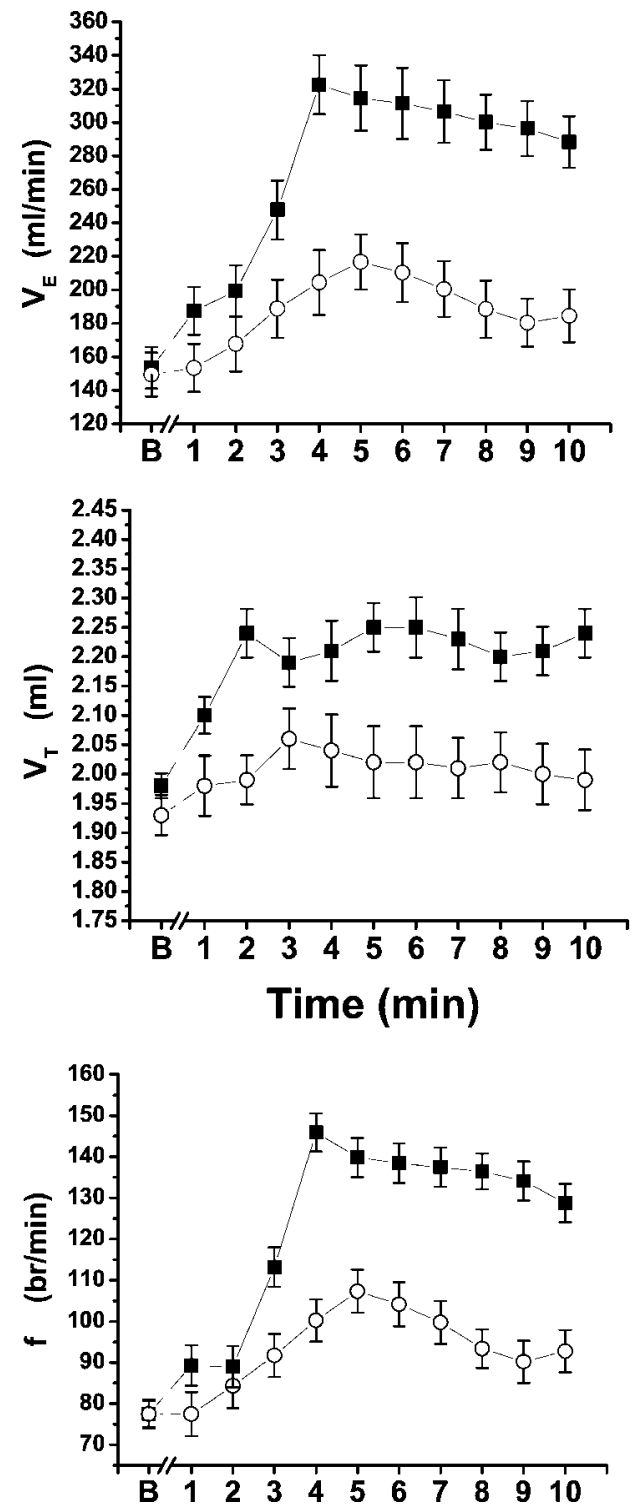

Figure 1. Mean $( \pm \mathrm{SD})$ minute ventilation $\left(V_{\mathrm{E}}\right)$, tidal volume $\left(V_{\mathrm{T}}\right)$, and respiratory frequency $(f)$ during normoxia $(B)$ and during every minute of a 10 min hypoxic challenge $\left(10 \% \mathrm{O}_{2}\right)$ in rats microinjected with either $1 \mathrm{~mm}$ domoic acid $(\bigcirc)$ or vehicle $(\mathbf{\square}) . n=10$ per group; DA versus vehicle; $p<0.0001$.

mmHg ( $p$ not significant). Of note, the effect of DA injection was also significantly different from the HVR changes occurring after vehicle was injected (Fig. 1) $(p<0.0001$; ANOVA). Rectal temperatures during normoxia and at the end of hypoxia were similar in DA- and vehicle-treated rats ( $p$ not significant).

In contrast to the HVR effects, DA treatment did not modify hypercapnic ventilatory responses to $5 \% \mathrm{CO}_{2}$ ( $p$ not significant).

\section{Peripheral chemoreceptor function after DA injections}

The mean ventilatory responses to bolus intravenous injections of $\mathrm{NaCN}$ are shown for both DA- and vehicle-treated rats (Fig. 3) ( $n=6$ per group). Ventilatory enhancements were markedly reduced in DA-treated rats, compared with controls $(p<$ 0.00001 ; ANOVA). However, the ventilatory strategy, as evidenced by changes in tidal volume and respiratory frequency in response to $\mathrm{NaCN}$, was unaffected in DA-treated animals.

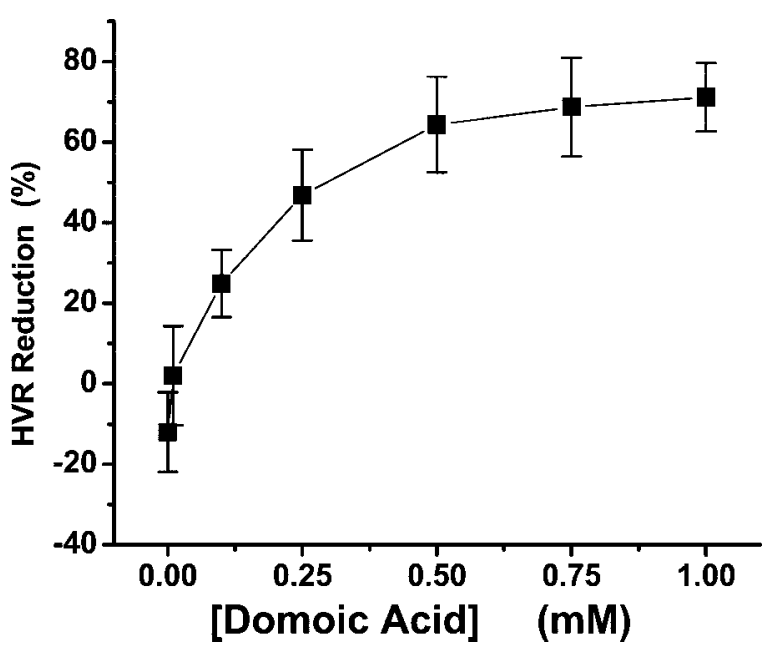

Figure 2. Mean $( \pm \mathrm{SD})$ attenuation of the hypoxic ventilatory response [expressed as percentage from baseline hypoxic ventilatory response $(H V R)]$ in rats microinjected with increasing doses of domoic acid in the nucleus of the solitary tract. A dose-dependent effect is apparent $(p<$ 0.001 ) and reaches a plateau at a domoic acid concentration of $0.75 \mathrm{~mm}$. $n=6$ per group.

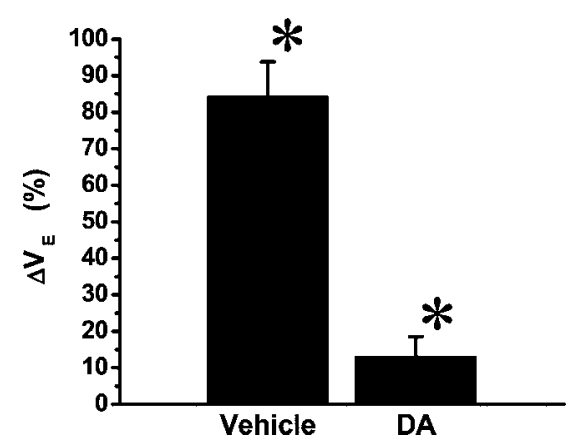

Figure 3. Changes in minute ventilation $\left(\Delta V_{\mathrm{E}}\right)$ after bolus intravenous injections of $40-80 \mu \mathrm{g} \mathrm{KCN}$ in rats microinjected with domoic acid or vehicle in the nucleus of the solitary tract. $n=6$ per group; ${ }^{*} p<0.00001$; ANOVA. ( $\Delta V_{\mathrm{E}}$ is expressed as percentage of prechallenge baseline values.)

\section{Baroreceptor function and gain after DA injections}

Acute intravenous injections of phenylephrine $(40 \mu \mathrm{g} / \mathrm{kg})$ were associated with significant and similar increases in systemic blood pressure in both DA- and vehicle-treated rats compared with bolus saline injections ( $p$ not significant). These pressor responses were qualitatively similar in the two treatment groups during anesthesia and during wakefulness conditions and were associated with similar reductions in $\operatorname{HR}(-35 \pm 4$ and $-33 \pm 4$ bpm; $p$ not significant). Because the responses to rapidly administered injections may prevent identification of more subtle changes in baroreceptor gain, dose-response curves were built across a wider range of blood pressure values for each animal by slow continuous infusion of phenylephrine and sodium nitroprusside (Fig. 4). As shown in Figure 4, the average baroreceptor gain derived from such experiments was similar between DA- and vehicle-treated animals. Indeed, mean gain in DA-treated animals was calculated at $3.72 \pm 0.22 \mathrm{bpm} / \mathrm{mmHg}$ and at $3.88 \pm 0.17$ $\mathrm{bpm} / \mathrm{mmHg}$ in controls ( $p$ not significant), and no shifts in the sigmoid curve occurred after DA treatment. 

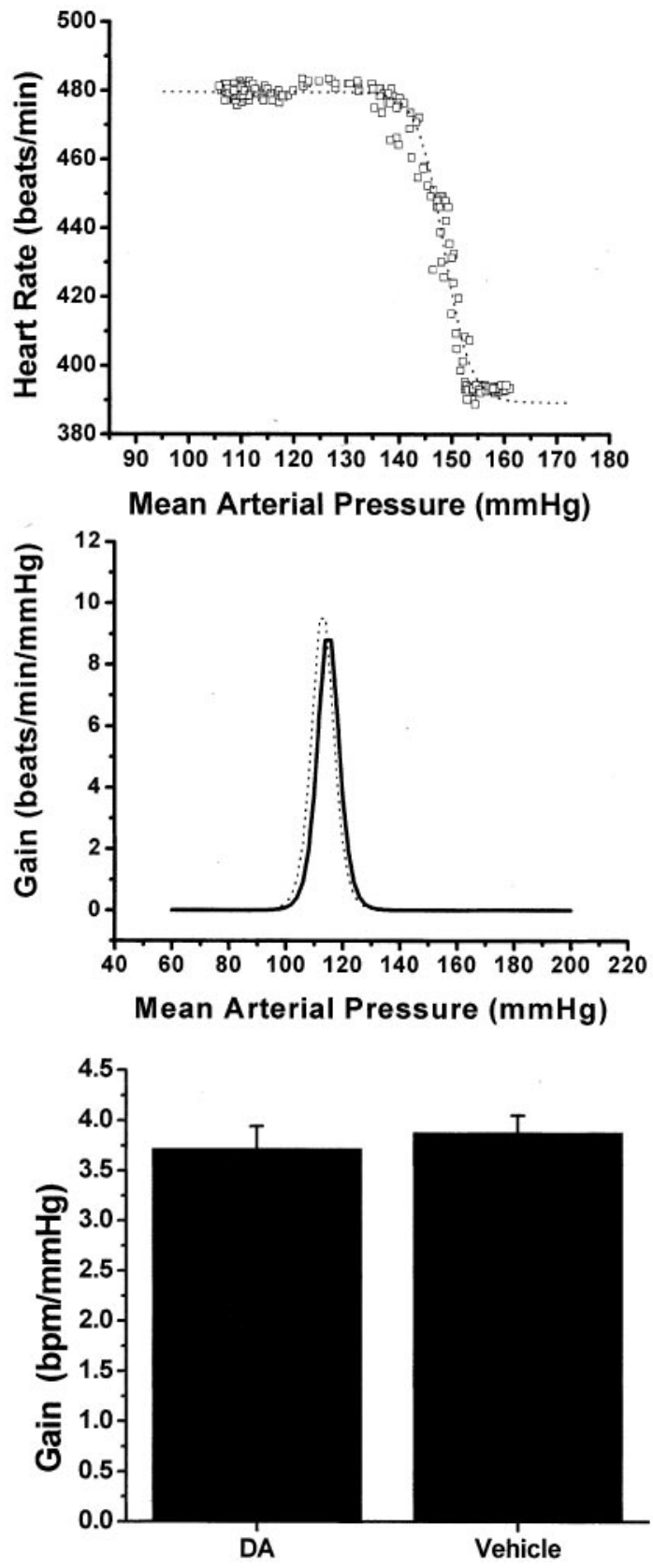

Figure 4. Top panel, Individual dose-response curve to blood pressure changes induced by intravenous infusions of either phenylephrine or sodium nitroprusside. The dotted line represents the best fit of a logistic regression function (see Materials and Methods). Middle panel, Individual gains derived from a logistic regression function in a rat previously microinjected with domoic acid in the nucleus of the solitary tract (dotted line) and a rat injected with vehicle (solid line). Bottom panel, Mean gain (expressed in beats per minute/millimeters mercury, pressure) of rats microinjected with domoic acid or vehicle. $n=8$ per group; $p$ not significant.

\section{Anatomical studies}

$D A$ injections in the NTS: anatomical verification by dual labeling

To determine the brainstem location and the area covered by DA injections, we used a double fluorescent labeling strategy using TMR-D as the anterograde tracer (red) and DiA (yellow-green) to mimic the DA injection procedure. The projection field of the vagal-glossopharyngeal afferent nerves is defined as the area

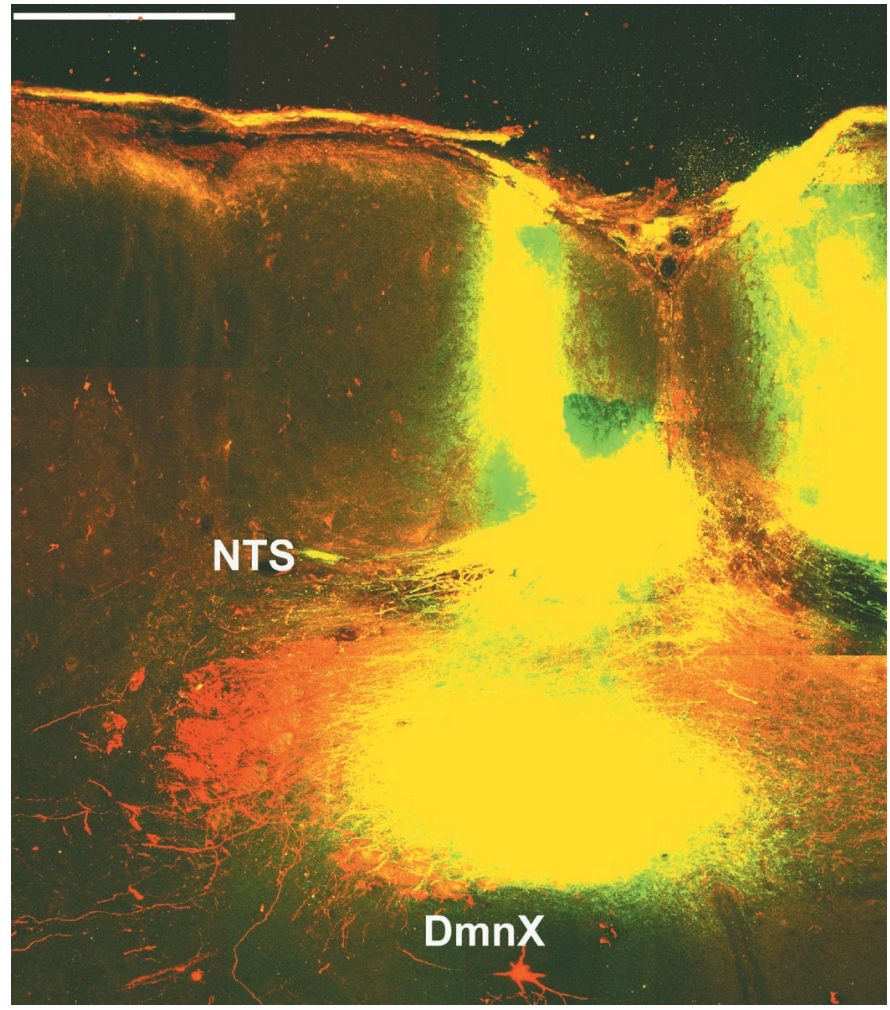

Figure 5. Confocal fluorescence photomicrograph of a section of the brainstem caudal to the obex illustrating the distribution of microinjections of the green-yellow tracer DiA. In the background and greatly overlapping with DiA ( green-yellow areas), labeling of unilateral peripheral afferent terminals in the NTS and DmnX was previously conducted by injection of the red anterograde tracer tetramethylrhodamine dextran into the left nodose ganglion. Scale bar, $340 \mu \mathrm{m}$.

innervated by the red TMR-D-labeled fibers and terminals. After DiA injections into the brainstem (mimicking DA injections), the DiA drops (green-yellowish) were found right at the centers of the NTS at caudal, middle, and rostral levels and covered a major portion of the NTS. However, the NTS was not completely covered by DiA drops. As shown in Figure 5, the green-yellowish drops did not cover all red fibers and terminals. An overall examination of the medullas of the four doubly injected animals indicated that the injection sites of DiA (green-yellowish) tended to be fused longitudinally in the dorsal vagal complex, including the NTS and the dorsal motor nucleus of the vagus. In addition, the central cores of DiA injections were centered at the NTS at most frontal levels, which were outlined by massive red afferent terminals, and the injection spheres extended ventral to the dorsal motor nucleus of the vagus only minimally. These experiments demonstrate that DA was injected precisely into the NTS and that the DA injections effectively covered the majority of the NTS region, albeit while leaving some small NTS areas uncovered.

\section{NTS cellular loss after DA lesions}

Examination of the cupric silver-stained brainstem slices revealed significant cellular losses in the NTS region. Figure $6 \mathrm{~A}$ is a section at the level caudal to the obex, and it shows the debris of the degenerating neurons in the commissural subnucleus. Figure $6 B$ is the control on the same glass slide showing the absence of the cupric silver staining in the commissural NTS in a salineinjected animal. Degenerating neurons were also abundant in other brainstem sections (data not shown). Therefore, DA lesions 

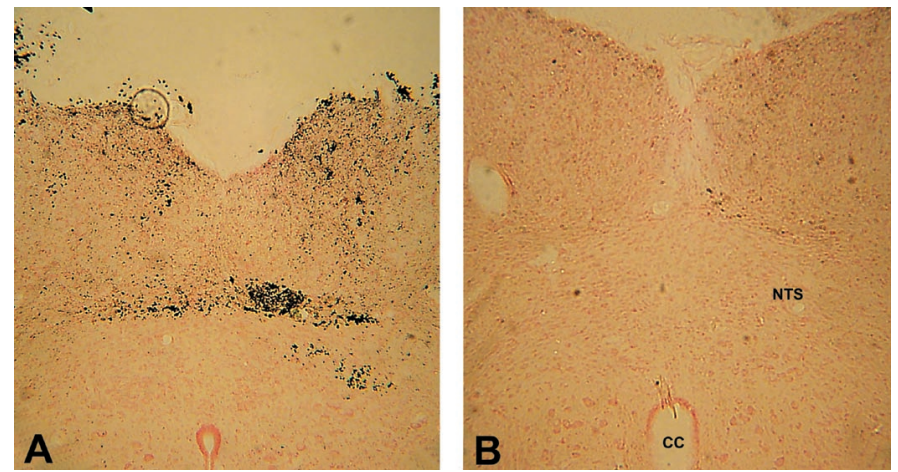

Figure 6. Photomicrographs of brainstem sections after cupric acid staining (black grains) for neuronal cell body degeneration in a rat microinjected with domoic acid $(A)$ and another microinjected with saline $(B) . c c$, Central canal.

led to significant losses of NTS neurons that were not present after vehicle injections.

\section{Degeneration of vagal-glossopharyngeal afferent terminals in DA-lesioned NTS}

In addition to the cellular loss of NTS neurons, afferent terminals in the NTS region were significantly decreased throughout the first month of DA injections. Figures $7 A, 8 A$, and $9 A$ are three montages of 3-D all-in-focus projection images of the afferent fibers and endings in the caudal, middle, and rostral NTS of a vehicle-treated animal, whereas Figures $7 B, 8 B$, and $9 B$ are the three corresponding montages from a representative DA-injected animal. These images clearly demonstrate that DA injections significantly reduced the afferent terminal fields and density in the caudal and middle NTS. The reduction of terminal fields and density in the rostral NTS sections was not as obvious as that seen in the caudal and middle sections, and this gradually rostrally evolving process showed that there were no significant differences in terminal fields and density at $+800 \mu \mathrm{m}$. Although all-in-focus projection montages usually present a reliable representation of the projection fields and overall density, more subtle differences in terminal ending density in some of the subregions of the NTS might not be apparent in the confocal all-in-focus projection images, because overlays of optical sectioned images on the top of each other could mask the details. To further explore this issue, we compared the corresponding individual optical sections sequentially. Figure 10 is a representative example that displays such comparisons. Figure $10 A$ is the image excerpt from the commissural subnucleus in Figure $7 A$ at higher magnification, and Figure $10 A^{\prime}$ is the seventh optical section of Figure $10 A$, whereas Figure $10 B$ corresponds to the image excerpt from the commissural subnucleus in Figure $7 B$, and Figure $10 B^{\prime}$ is the seventh optical section of Figure $10 \mathrm{~B}$. When such comparisons were performed, the extent and density of afferent terminals in the commissural NTS of DA-treated animals were greatly reduced and sparser than in those derived from control animals. Figure 7, $A$ and $B$, were composed of 14 optical sectioned images, and comparisons were made across all corresponding individual optical sections. In addition to the commissural subnucleus, the density of the afferent terminals in the dorsomedial NTS, which is dorsal to the DmnX, was also much sparser in the caudal and middle levels, as shown in Figures 7-9. Opening of the labeled codes resulted in perfect matching between the experimented group allocation and the actual DA and vehicle treatment groups.
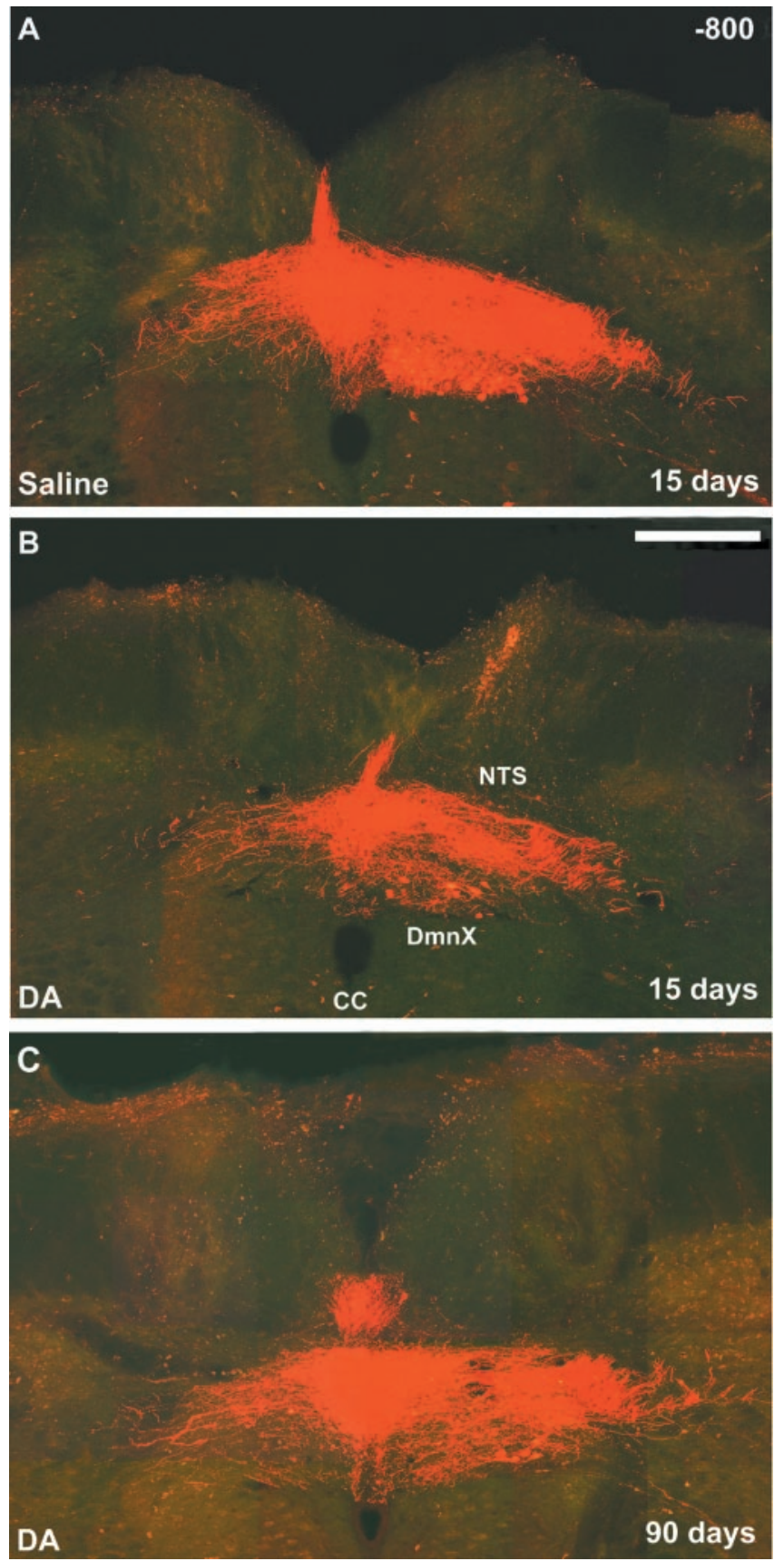

Figure 7. Confocal photomicrographs of brainstem sections $(-800 \mu \mathrm{m}$ from obex) after unilateral labeling of peripheral afferent terminals in the NTS and DmnX after injection of the right nodose ganglion with the red anterograde tracer tetramethylrhodamine dextran (TMR-D). $A$ corresponds to a rat microinjected with saline $15 \mathrm{~d}$ earlier, $B$ corresponds to a rat microinjected $15 \mathrm{~d}$ earlier with domoic acid $(1 \mathrm{~mm})$, and $C$ represents the same brainstem region imaged from a rat microinjected $90 \mathrm{~d}$ earlier with domoic acid. Note the substantial reduction of axonal projections within the NTS and the number of motor neurons in DmnX in $B$; similarly, note the prominent enhancement of axonal densities in NTS subnuclei spreading ventrally to the $\mathrm{DmnX}$ region and also dorsally and to the contralateral NTS in $C$ without any discernible changes in DmnX motor neuron population. Scale bar, $340 \mu \mathrm{m}$.

Twelve animals (six saline- and six DA-treated) were ranked according to their afferent terminal densities within the NTS by a blinded investigator. The six DA-treated animals had comparable afferent terminal densities, but all of them ranked differently from 

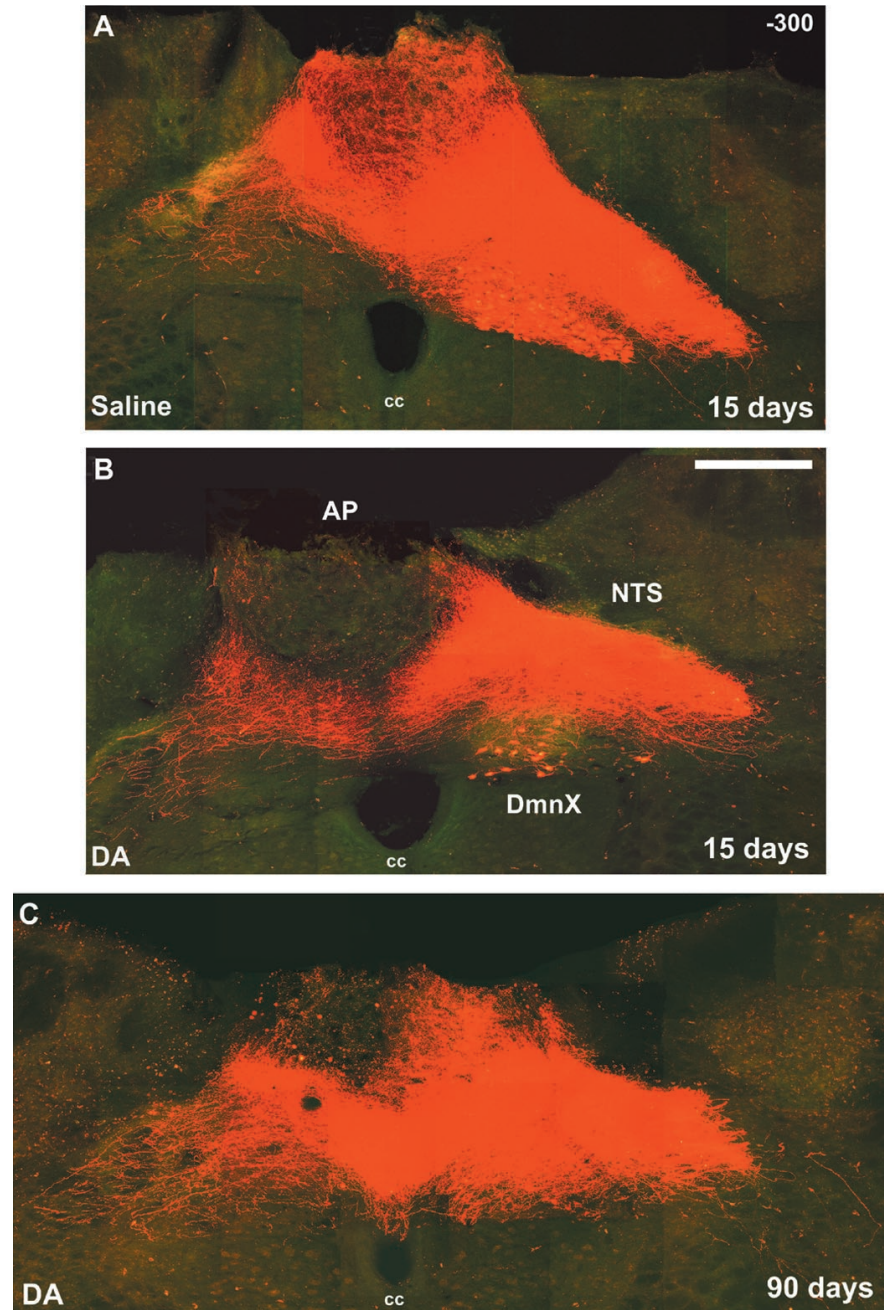

Figure 8. Confocal photomicrographs of brainstem sections [300 $\mu \mathrm{m}$ from obex at the level of area postrema $(A P)]$ after unilateral labeling of peripheral afferent terminals in the NTS and DmnX after injection of the right nodose ganglion with the red anterograde tracer TMR-D. $A$ corresponds to a rat microinjected with saline $15 \mathrm{~d}$ earlier, $B$ corresponds to a rat microinjected $15 \mathrm{~d}$ earlier with domoic acid $(1 \mathrm{~mm})$, and $C$ represents the same brainstem region imaged from a rat microinjected $90 \mathrm{~d}$ earlier with domoic acid. Note substantial reduction of axonal projections within the AP and the NTS, both ipsilaterally and contralaterally, and the decreased number of motor neurons in DmnX in $B$; similarly, note prominent enhancement of axonal densities in $C$ without any discernible changes in motor neuron population. Axonal proliferation/regeneration is evident in the commissural (ipsilateral and contralateral to injection side) and the dorsomedial regions of the NTS, the AP, and the DmnX region. Scale bar, $340 \mu \mathrm{m}$.

all of the six saline-treated animals, in whom terminal densities were much higher. Such reductions in DA-treated animals were not caused by insufficient tracer labeling in DA-treated animals. As shown below, the afferent terminals in the animals examined at $90 \mathrm{~d}$ after DA injection were much denser than those found $15 \mathrm{~d}$ after DA lesions and in fact were comparable to saline controls. In contrast, the number of DmnX neurons at $90 \mathrm{~d}$ was similar or even further reduced compared with $15 \mathrm{~d}$. Therefore, DA treatment led to a significant reduction of the projection fields and density of afferent fibers at 15-30 d after surgery.

\section{Reduction of motor neurons in the DmnX after DA injections}

In addition to the vagal afferent terminal loss in the NTS, the vagal motor neurons in DmnX also underwent substantial degen-
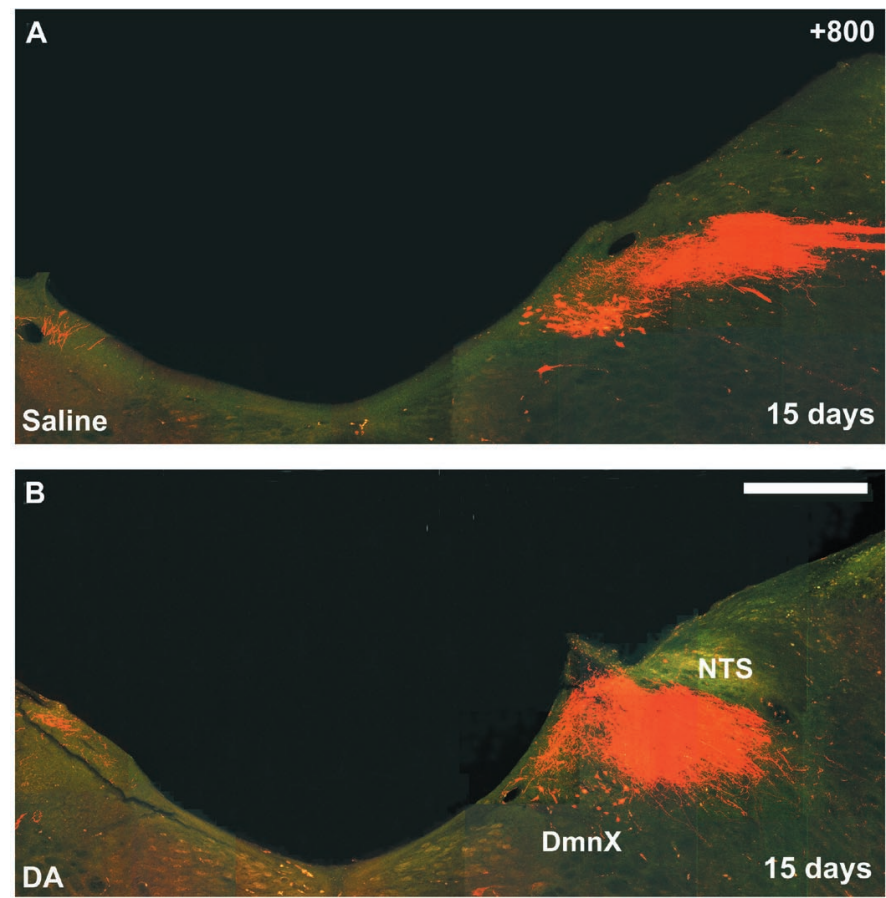

C

DA

90 days

Figure 9. Confocal photomicrographs of brainstem sections $(+800 \mu \mathrm{m}$ rostral to obex) after unilateral labeling of peripheral afferent terminals in the NTS and DmnX after injection of the right nodose ganglion with the red anterograde tracer TMR-D. $A$ corresponds to a rat microinjected with saline $15 \mathrm{~d}$ earlier, $B$ corresponds to a rat microinjected $15 \mathrm{~d}$ earlier with domoic acid $(1 \mathrm{~mm})$, and $C$ represents the same brainstem region imaged from a rat microinjected $90 \mathrm{~d}$ earlier with domoic acid. Note no obvious reduction of axonal projections within the NTS. However, the decreased number of motor neurons in DmnX is markedly present in $B$. In $C$, no changes are evident in axonal projections; however, the substantial reduction in motor neuronal pool of the DmnX persists. Scale bar, $340 \mu \mathrm{m}$.

eration. The number of retrogradely labeled motor neurons by TMR-B injection into the nodose ganglion was markedly reduced in all brainstem sections of DA-treated animals. Figure 11, $A$ and $B$, are image excerpts from DmnX regions in Figure 8, $A$ and $B$, at higher magnification. These figures clearly illustrate that the number of DmnX neurons in the DA-treated brainstem is much smaller than that of vehicle-treated animals. Comparisons between the corresponding DmnX regions of DA and control animals were made across all the brainstem sections of these two animals. In each brainstem section of DA-treated NTS, the number of DmnX neurons was usually $<10-15$. In contrast, there were numerous DmnX neurons in each section of vehicle-treated animals, and they were so densely compacted that accurate counts with the conventional epifluorescence microscope became impossible. However, the number of DmnX neurons after vehicle treat- 

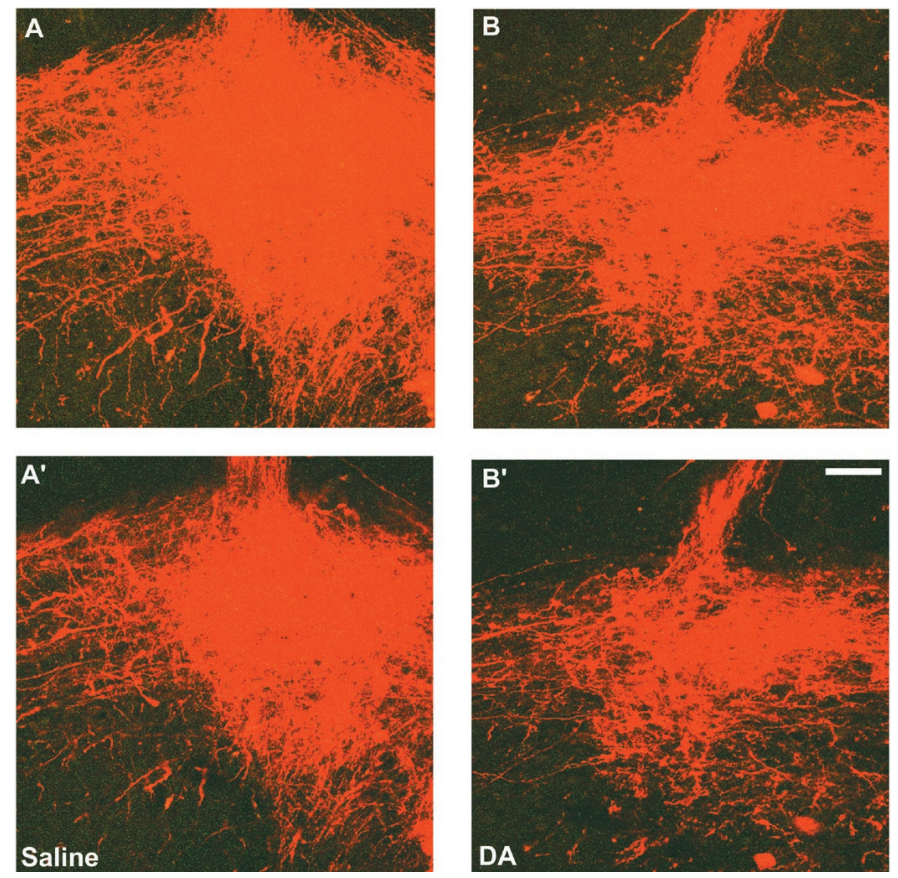

Figure 10. Photomicrographs of brainstem sections after unilateral labeling of peripheral afferent terminals in the NTS and DmnX after injection of the right nodose ganglion with the red anterograde tracer TMR-D. $A$ and $B$ represent higher magnifications (scale bar, $50 \mu \mathrm{m}$ ) of the confocal photomicrographs shown in Figure 7, $A$ and $B . A^{\prime}$ and $B^{\prime}$ represent single optical sections of the same region selected at the same stack hierarchical level. A reduction in the density of nerve terminal endings in the commissure is apparent in $B^{\prime}$ compared with $A^{\prime}$.

ment was $>50$ in all cases. Therefore, DA injection into the NTS was associated with marked reductions in the number of DmnX neurons.

\section{Functional recovery}

To examine the long-term functional effect of DA lesions, we measured peak HVRs in eight DA and eight vehicle-treated rats at 15, 30, 60, and $90 \mathrm{~d}$ (Fig. 12). Peak HVR was $182 \pm 23 \%$ from normoxic baseline at $1 \mathrm{~d}$ before surgery and was attenuated to $\sim 58 \pm 17 \%$ from corresponding normoxic baseline, therefore a reduction of $\sim 70 \%$ at $15 \mathrm{~d}$ after DA injection. At $30 \mathrm{~d}$, no significant changes in peak HVR had occurred $(p>0.05)$. However, HVR was $111 \pm 26 \%$ or $60.1 \%$ of the baseline and hence displayed a significant recovery at $60 \mathrm{~d}$ after DA $(p<0.01$ vs 15 and $30 \mathrm{~d}$ ), and by $90 \mathrm{~d}$, HVR was $176 \pm 28 \%$ or $96 \%$ of the baseline and thus was not significantly different from pretreatment control $(p>0.05)$, thereby indicating complete functional recovery. In contrast, no significant changes occurred over time in eight vehicle-treated animals. Therefore, complete functional recovery developed spontaneously within $90 \mathrm{~d}$ after DA-induced NTS lesions in adult rats.

\section{Axonal regeneration in the NTS}

At $97 \mathrm{~d}$ after surgery ( $7 \mathrm{~d}$ were necessary for retrograde tracer transport), significant afferent axonal sprouting was found in the NTS. Figures $7 C, 8 C$, and $9 C$ show three montages of the caudal, middle, and rostral brainstem sections. When compared with the findings obtained in DA- and saline-treated animals at $15 \mathrm{~d}$, several important differences emerged in animals after $90 \mathrm{~d}$. In caudal and middle sections, the regenerating afferent axons
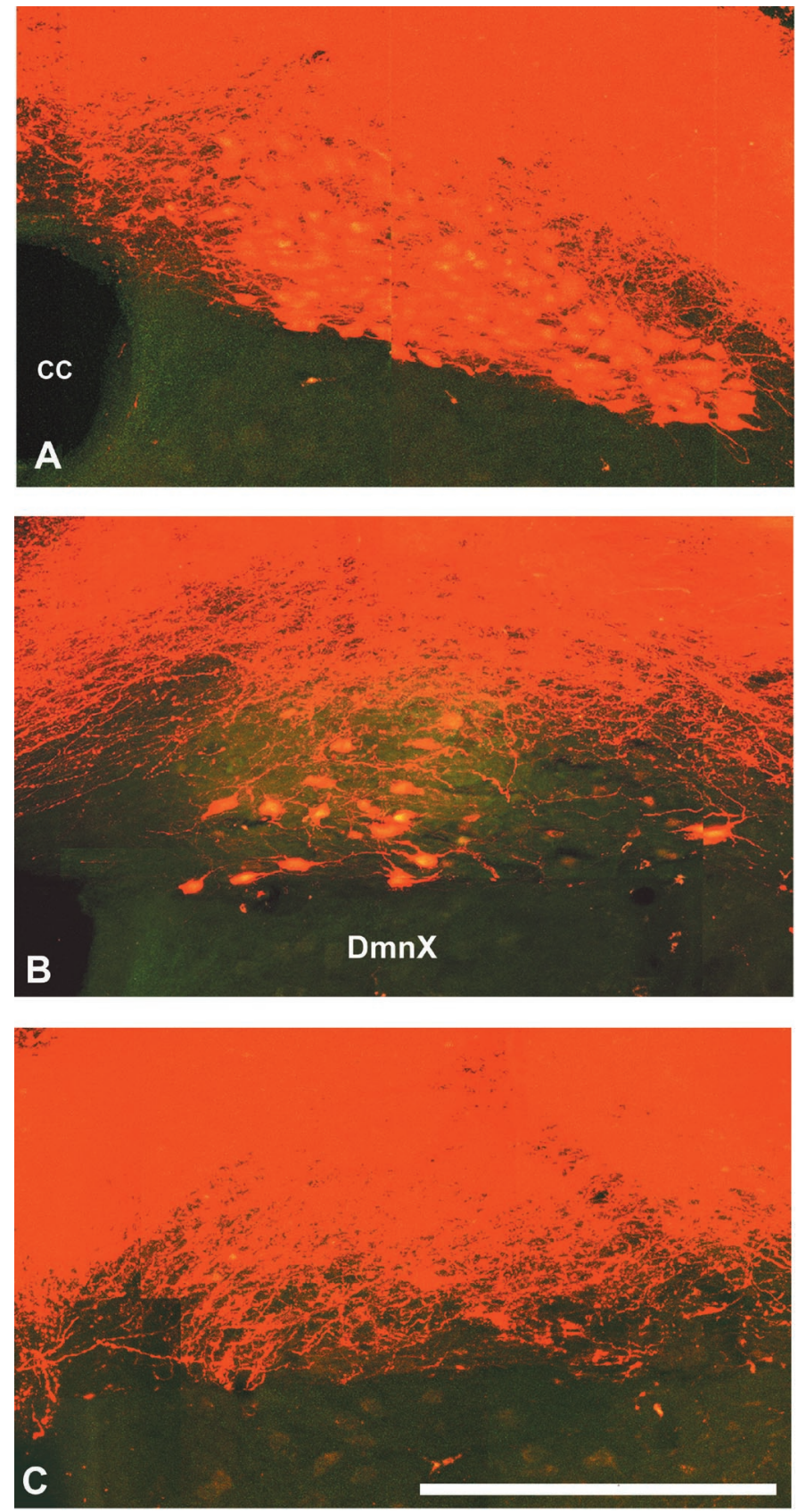

Figure 11. Confocal photomicrographs of brainstem sections $[-300 \mu \mathrm{m}$ from obex at the level of area postrema) after unilateral labeling of peripheral afferent terminals in the NTS and DmnX after injection of the right nodose ganglion with the red anterograde tracer TMR-D. These confocal images correspond to larger magnification of the DmnX regions imaged in Figure $8 A-C$ to illustrate the extensive loss of motor neurons after domoic acid microinjection at $15 \mathrm{~d}$, and the extensive regeneration of nerve terminals in the DmnX area after functional recovery occurs at 90 d. Scale bar, $340 \mu \mathrm{m}$.

sprouted into the field almost identical to the normal afferent fiber projection field in the NTS. Particularly, the commissural subnucleus, which is the major relay station for primary chemoreceptor fibers, was refilled densely with the regenerating axons. In addition, the density of terminals in the contralateral brainstem was increased. Although axonal sprouting was also found in the area postrema, the density of the regenerating terminals was reduced compared with the axonal projection densities in control 


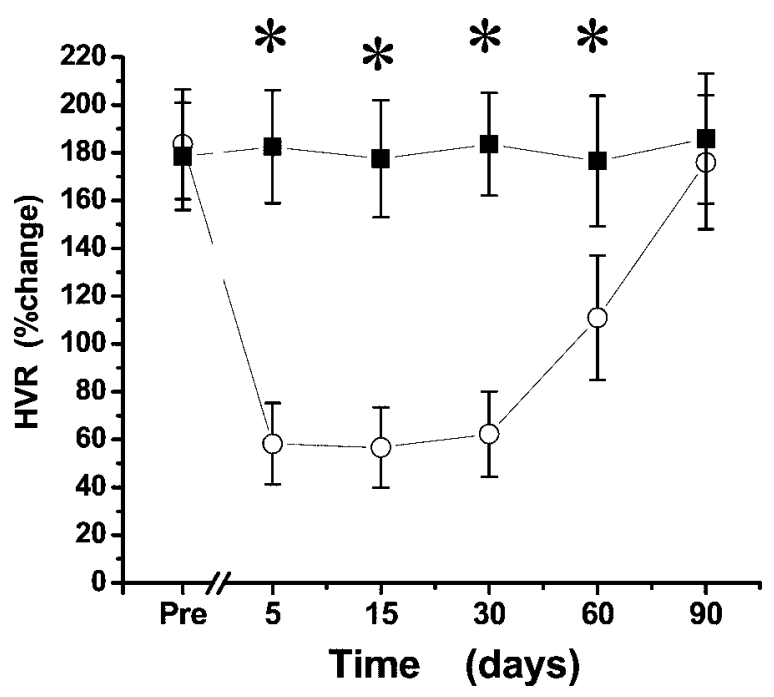

Figure 12. Changes in HVR over time in rats microinjected with domoic acid $(\bigcirc)$ or vehicle $(\mathbf{\square})$. Significant attenuation of HVR after 5, 15, 30, and $60 \mathrm{~d}$ is present after DA treatment compared with vehicle $\left({ }^{*} p<\right.$ 0.00001 ; ANOVA; $n=8$ per group). However, recovery is already apparent at $60 \mathrm{~d}$ and is complete $90 \mathrm{~d}$ after DA microinjection.

rats. Therefore, spontaneous peripheral afferent axonal sprouting occurred in both density and distribution patterns at $90 \mathrm{~d}$ after DA lesions of the NTS in adult rats and was not found at $30 \mathrm{~d}$.

\section{DmnX motor neurons after functional recovery}

No regeneration of DmnX neurons was observed after $90 \mathrm{~d}$ after DA injections. Compared with the number of DmnX neurons observed in rats at $15 \mathrm{~d}$ after DA treatment, the number of DmnX neurons was either unchanged or in two cases reduced even further, as shown in Figures $7 C, 8 C$, and $9 C$ and Table 1 . Interestingly, the regenerating axons dipped down into the DmnX, entered the empty areas left by the lost DmnX neurons, and sprouted, as shown in Figures $7 C, 8 C$, and $9 C$.

\section{DISCUSSION}

The present study shows that bilateral DA microinjections lead to substantial attenuation of hypoxic ventilatory responses in waking rats lasting $30 \mathrm{~d}$, consistent with cellular loss of NTS neurons, and reduction of vagal-glossopharyngeal afferent terminals in the NTS. However, in the subsequent $60 \mathrm{~d}$, spontaneous functional recovery occurs and parallels afferent terminal sprouting, particularly in the commissural and dorsomedial NTS. Therefore, the NTS is not only critical for normal respiratory reflexes but has self-repairing capability, i.e., functional recovery plasticity is possible in brainstem neural sites. Thus, this study provides a useful model for the future study of mechanisms underlying the functional and anatomical plasticity of the NTS.

\section{Domoic acid lesion approach}

The anatomical and functional evidence suggest that the DA lesion approach eliminated the majority of the glutamate receptor neurons in the NTS but left others intact. However, there are two concerns about the completeness of DA lesions in the NTS. First, the NTS is a relatively large nucleus. Therefore, it is a valid question as to whether the DA injections covered the whole NTS. Second, DA has relatively higher affinity to glutamate AMPA than NMDA receptors (Hampson et al., 1998). Therefore, it is questionable whether DA might be sufficient to kill all targeted neurons expressing NMDA receptors in the NTS. Nevertheless, three lines of evidence support the idea that DA was able to destroy the majority of NMDA neurons in the NTS, albeit leaving some neurons intact. First, we used double-labeling strategy to mimic the extent of DA injection sites in the entire NTS rostrocaudally. The cores of yellow-green dye DiA injection were right at the NTS region and tended to cover most of the red TMR-labeled afferent terminals in the NTS. Also, the cores of DiA injections tended to fuse together longitudinally with the cores of the next DiA injections in the subsequent sections. Considering that these doubly labeled brainstems were taken immediately after DiA injections and hence the spread of DiA into the NTS would be minimal, we assume that the area covered by $\mathrm{DiA}$ injections should be much smaller than the real area affected, at a time when presumably DA had already spread to a wider area of the NTS. However, some chemoreceptor-sensitive neurons might have escaped from being destroyed. In fact, as shown in Figure 5, although the DiA injection covered a large zone of the NTS, some other areas innervated by the red afferent terminals were not covered. Second, we explored the dosedependency effects of DA. The slope of the HVR reduction curve became smaller at $>0.5 \mathrm{~mm}$ and increasing the dose did not further decrease HVR. It is conceivable, however, that even higher doses or alternatively increasing the number of DA injection sites might be able to lesion more neurons and induce further reductions of the HVR. Unfortunately, we were unable to test this possibility because DA at $>1 \mathrm{~mm}$ did permit animal recovery. Third, because carotid sinus nerve denervation leads to $\sim 70 \%$ HVR reductions, and addition of cervical trunk vagotomy completely eliminates the HVR (Ohtake et al., 1998), we suggest that the neurons destroyed by DA would contribute to $\sim 70 \%$ of the HVR in normal physiological conditions and that surviving neurons may provide the neural network mediating the residual HVR. Therefore, we conclude that our approach effectively destroyed the majority of NTS neurons.

\section{Cardiovascular effect of DA lesions in the NTS}

The absence of readily identifiable effects of DA-induced NTS lesions on long-term baroreceptor function was surprising. These regions of the NTS have long been implicated in mediating important components of cardiovascular function (West et al., 1981; Sved, 1986; Andresen and Kunze, 1994; Aylwin et al., 1997; Zhang and Mifflin, 1998; Machado, 2001). However, most of these studies involved nonsurvival experiments, such that only the acute effects of lesions were determined. Furthermore, the preserved integrity of other pressor and depressor regions within the brainstem may be sufficient for adaptive regulatory mechanisms of blood pressure regulation. Indeed, Gieroba and Blessing (1992) have shown that neurons in the caudal ventrolateral medulla, which inhibit sympathetic vasomotor tone and have reciprocal connections to the NTS, do not require NTS integrity to exert their pressor and depressor effects. Similarly, Sato et al., (1999) showed that in contrast with acute NTS lesions, chronic NTS lesions do not modify blood pressure responses to bilateral carotid occlusion maneuvers. Thus, reorganization of the remaining aortic baroreceptors or of the structures underlying baroreflex function may result in normalization of the cardiovascular responses after NTS lesions. Alternatively, as inferred from the differential disruption of axonal projections along the rostrocaudal axis of the NTS, it is possible that more rostrally located neurons have reduced susceptibility to DA injections.

DmnX motor neuron loss did not affect the beat-by-beat 


\begin{tabular}{|c|c|c|c|c|}
\hline & Caudal $(-800 \mu \mathrm{m})$ & Middle $(-300 \mu \mathrm{m})$ & Rostral $(+800 \mu \mathrm{m})$ & Total \\
\hline Saline & $41 \pm 5$ & $133 \pm 12$ & $56 \pm 7$ & $230 \pm 16$ \\
\hline $15 \mathrm{~d}$ & $7 \pm 1(17.1 \%)$ & $18 \pm 3(13.5 \%)$ & $12 \pm 3(21 \%)$ & $37 \pm 5(16.1 \%)$ \\
\hline $90 \mathrm{~d}$ & $6 \pm 1(14.6 \%)$ & $6 \pm 1(4.5 \%)$ & $1 \pm 1(1.7 \%)$ & $13 \pm 3(5.7 \%)$ \\
\hline
\end{tabular}

The distances from the obex are shown in parentheses; $(\%)$ indicates the reduction compared with the saline controls; $n=3$ per group.

baroreflex at $15 \mathrm{~d}$ after the DA lesion, indicating that the DmnX may not be a major nucleus for baroreceptor function in this chronically lesioned model. However, the acute cardiorespiratory effects of the DmnX motor neurons during the DA injection were not tested. Because anatomical data from our laboratory strongly indicate that the DmnX cardiac motor neurons project to cardiac ganglions (Cheng et al., 1999), the acute excitotoxic death of DmnX motor neurons is likely to affect the baroresponse in the acute phase after DA injection. Indeed, the NA is the predominant nucleus innervating cardiac ganglia (Cheng and Powley, 2000) and plays a more significant role in controlling the cardiac functions (Loewy and Spyer, 1990). Thus, we speculate that either NA cardiac neurons compensated for the functional loss of the DmnX cardiac neurons or that the DmnX motor neurons may have different modulatory cardiorespiratory functions or be involved in cardiac reflexes elicited by pulmonary afferent fiber activation (Jones et al., 1995, 1998; Cheng et al., 2000; Wang et al., 2000).

\section{Functional ventilatory recovery}

This study demonstrates for the first time that complete respiratory functional recovery may occur after extensive damage to the NTS in adult rats. Although our study does not provide any insights on the mechanism(s) involved in such spontaneous recovery, we propose four nonmutually exclusive possibilities that might have contributed to this functional recovery. (1) First, centrally located neurons functioning as $\mathrm{O}_{2}$ sensors, i.e., increasing discharge firing with hypoxia, may have assumed an increased role in the functional components of the HVR. Indeed, several brain areas exhibit $\mathrm{O}_{2}$ sensing properties and can function independently from peripheral chemoreceptor inputs. For example, in the adult isolated brainstem preparation, hypoxia will modify respiratory activity as recorded from the hypoglossal nerve rootlet (Schafer et al., 1993). Similarly, caudal hypothalamic $\mathrm{O}_{2}$ sensing neurons can facilitate the respiratory response to hypoxia independently of peripheral chemoreceptors (Horn and Waldrop, 1997). Moreover, brainstem neurons located in cardiorespiratory control areas may exhibit intrinsic $\mathrm{O}_{2}$ sensitivity (Sun and Reis, 1994; Nolan and Waldrop,1996). Kawai et al. (1999) further identified two different populations of RVLM neurons that can be directly stimulated by hypoxia. However, these findings were derived from studies conducted in reduced preparations, and the functional role of these oxygen-sensing neurons remains unknown in the awake, freely behaving animal. (2) Plasticity and reorganization of the respiratory network can occur after removal of peripheral chemoafferent input, indicating a gain of function mediated through central brain structures assuming the role previously played by carotid body inputs. Indeed, Roux et al. (2000a,b) provided compelling evidence that after irreversible bilateral carotid sinus nerve transection in the unrestrained adult rat, the HVR attenuation elicited by this procedure $(\sim 70 \%)$ was fully recovered within 6 weeks and was associated with a profound functional reorganization of the central $\mathrm{O}_{2}$ chemoreflex pathway, suggesting that plasticity changes in centrally located neural networks were primarily responsible for the functional recovery. However, Roux et al. (2000a,b) reached such conclusions on the basis of the assumption that peripheral noncarotid chemoreceptors have no functional role in HVR. Similar to the aortic nerves, the functions of other noncarotid peripheral chemoreceptor fibers are still debatable (Cheng et al., 1997b, Kobayashi et al., 1999; Forster et al., 2000). Forster et al. (2000) studied neonatal piglets and showed that during the first few days of life chemosensitivity was present in both carotid and aortic chemoreceptor areas. When the carotid chemoreceptors were removed early in life, the aortic chemoreceptors provided enough stimulation to prevent hypoventilation. However, with intact carotid chemoreceptors, the aortic chemoreceptors eventually became nonf unctional. These findings suggest that peripheral noncarotid chemoreceptors, which in normal conditions play a minor and redundant role in HVR, can display a gain of function in response to injury. In our lesion experiments, the acute effects on HVR were strikingly similar to those elicited by bilateral deafferentation. Indeed, HVR was $30 \%$ of control in all cases (Ohtake et al., 1998; Roux et al., 2000a,b). However, the time course of the functional recovery after DA lesioning of the NTS was slower (90 vs $21 \mathrm{~d}$ ). Thus, we have to assume that both peripheral noncarotid chemoreceptors and central $\mathrm{O}_{2}$ sensors contributed to the residual HVR and to the functional recovery processes in both models. (3) A third possibility may involve the upregulation of residual NMDA transmission in the NTS. As discussed previously, DA injections may have left a small portion of viable NTS neurons. Thus, it is possible that undamaged neurons within a given structure may upregulate their function and compensate for the functional loss caused by the lesion. For example, Weidner et al. (2001) recently showed that after complete lesions of the dorsal corticospinal motor pathways, which contains $>95 \%$ of all ventral corticospinal axons, spontaneous sprouting from the ventral corticospinal tract occurred within medial motor neuron pools of the cervical spinal cord. This sprouting was correlated with full functional recovery. Thus, the intact ventral corticospinal tract exhibited a gain of function and assumed the role usually played by the large dorsal corticospinal tract. Similarly, Chan et al. (2000) induced bilateral carotid sinus nerve lesions and showed that the aortic depressor nerves may be upregulated to enhance baroreceptor inputs to the NTS, as evidenced by a restoration of the normal pattern of Fos induction in responses to phenylephrine infusion after $30 \mathrm{~d}$ recovery. Therefore, increased expression of NMDA receptors and changes in the subunit composition of the residual NMDA receptors may have contributed to the functional recovery. (4) Finally, the correlation between functional recovery and the emergence of peripheral afferent axonal sprouting may argue for a mechanism involving the self-repairing capability of the adult CNS. Magavi et al. (2000) showed that neurogenesis may occur in the brain areas of adult mice that do not normally undergo any neurogen- 
esis. Indeed, induction of apoptotic degeneration of corticothalamic neurons in layer VI of anterior cortex of adult mice was followed 2-28 weeks later by the appearance of new neurons in this layer (Magavi et al., 2000). The newly formed neurons appeared to be recruited from the resident cortical progenitors or from the underlying subventricular zone in the wall of the lateral ventricle, or both, and some of these cells seemed to have connections to remote regions such as the thalamus. These exciting findings suggest that neural progenitors may migrate to the injury sites and under specific conditions may replace the missing neurons and presumably recover loss of function. It is conceivable that after DA-mediated neural injury, progenitor cells might have migrated to the NTS, generated new neurons, and established a functional circuitry by connecting to regenerating afferent axons. At this preliminary stage, we can only speculate that after the DA lesion, disconnected vagal axons may initially retract and then regenerate and sprout to innervate surviving or newly forming neurons, whereas parallel changes within non-NTS-located central $\mathrm{O}_{2}$-sensing neurons may also contribute to the functional recovery process. Notwithstanding such considerations, the current findings clearly justify future efforts to characterize the neural mechanism(s) underlying the functional recovery.

\section{Conclusions}

The present experiments conclusively demonstrate that the integrity of the NTS is critically important for the HVR, as evidenced by $70 \%$ HVR reductions immediately after DA-induced lesions that extensively reduce the density of vagal afferent projections to the nucleus. Contrasting with the conventional concept that suggests that damages to the adult CNS in general, and more specifically damage to brainstem structures, may lead to permanent functional deficits, our experiments demonstrate that the HVR can spontaneously and fully recover within $90 \mathrm{~d}$ after DAinduced NTS lesions. This recovery coincides with the growth of vagal afferent terminals within the NTS, particularly within the commissural nucleus, the primary region for chemoreceptor afferent input. Experiments aiming to examine cellular mechanisms potentially underlying the functional and structural recovery reported herein are under way in our laboratory.

\section{REFERENCES}

Andresen MC, Kunze DL (1994) Nucleus tractus solitarius: gateway to neural circulatory control. Annu Rev Physiol 56:93-116.

Aylwin ML, Horowitz JM, Bonham AC (1997) NMDA receptors contribute to primary visceral afferent transmission in the nucleus of the solitary tract. J Neurophysiol 77:2539-2548.

Bartlett DJB, Tenney SW (1970) Control of breathing in experimental anemia. Respir Physiol 10:384-395.

Björklund A, Lindvall O (2000) Neurobiology: self-repair in the brain. Nature 405:892-893.

Buchholz RA, Nathan MA (1984) Chronic lability of the arterial blood pressure produced by electrolytic lesions of the nucleus tractus solitarii in the rat. Circ Res 54:227-234.

Chan RKW, Jarvina EV, Sawchenko PE (2000) Effects of selective sinoaortic denervations on phenylephrine-induced activational responses in the nucleus of the solitary tract. Neuroscience 101:165-178.

Cheng Z, Powley TL (2000) Nucleus ambiguus projections to cardiac ganglia of rat atria: an anterograde tracing study. J Comp Neurol 424:588-606.

Cheng Z, Powley TL, Schwaber JS, Doyle III FJ (1997a) Vagal afferent innervation of the atria of the rat heart reconstructed with confocal microscopy. J Comp Neurol 381:1-17.

Cheng Z, Powley TL, Schwaber JS, Doyle IIIFJ (1997b) A laser confocal microscopic study of vagal afferent innervation rat aortic arch: chemoreceptors as well as baroreceptors. J Auton Nerv Syst 67:1-14.

Cheng Z, Powley TL, Schwaber JS, Doyle III FJ (1999) Projections of the dorsal motor nucleus of the vagus to cardiac ganglia of rat atria: an anterograde tracing study. J Comp Neurol 410:320-341.

De Caro R, Parenti A, Montisci M, Guidolin D, Macchi V (2000) Solitary tract nuclei in acute heart failure. Stroke 31:1187-1193.
Derrick M, He J, Brady E, Tan S (2001) The in vitro fate of rabbit fetal brain cells after acute in vivo hypoxia. J Neurosci 21:1-5.

Drorbaugh JE, Fenn WO (1955) A barometric method for measuring ventilation in newborn infants. Pediatrics 16:81-87.

Epstein RA, Epstein MAF, Haddad GG, Mellins RB (1980) Practical implementation of the barometric method for measurement of tidal volume. J Appl Physiol 49:1107-1115.

Fix AS, Ross JF, Stitzel SR, Switzer RC (1996) Integrated evaluation of central nervous system lesions: stains for neurons, astrocytes, and microglia reveal the spatial and temporal features of MK-801-induced neuronal necrosis in the rat cerebral cortex. Toxicol Pathol 24:291-304.

Forster HV, Pan LG, Lowry TF, Serra A, Wenninger J, Martino P (2000) Important role of carotid chemoreceptor afferents in control of breathing of adult and neonatal mammals. Respir Physiol 119:199-208.

Gieroba ZJ, Blessing WW (1992) Effect of nucleus tractus solitarius lesions on cardiovascular responses elicited from the caudal ventrolateral medulla. J Auton Nerv Syst 39:97-104.

Goldberg JL, Barres BA (2000a) Neurobiology: No go in nerve regeneration. Nature 403:369-370.

Goldberg JL, Barres BA (2000b) The relationship between neuronal survival and regeneration. Annu Rev Neurosci 23:579-612.

Gozal D (1998) Sleep-disordered breathing and school performance in children. Pediatrics 102:616-620.

Gozal D, Torres JE, Gozal YM, Littwin SM (1996a) Effect of nitric oxide synthase inhibition on cardiorespiratory responses in the conscious rat. J Appl Physiol 81:2068-2077.

Gozal D, Gozal E, Gozal YM, Torres JE (1996b) Nitric oxide synthase isoforms and peripheral chemoreceptor stimulation in conscious rats. NeuroReport 7:1145-1148.

Gozal D, Daniel JM, Dohanich GP (2001) Behavioral and anatomical correlates of chronic episodic hypoxia during sleep in the rat. J Neurosci 21:2442-2450.

Guyenet PG (2000) Neural structures that mediate sympathoexcitation during hypoxia. Respir Physiol 121:147-162.

Hampson DR, Manalo JL (1998) Activation of glutamate receptors by kainic acid and domoic acid. Nat Toxins 6:153-158.

Horn EM, Waldrop TG (1997) Oxygen-sensing neurons in the caudal hypothalamus and their role in cardiorespiratory control. Respir Physiol 110:219-228.

Housley GD, Sinclair JD (1988) Localization by kainic acid lesions of neurones transmitting the carotid chemoreceptor stimulus for respiration in rat. J Physiol (Lond) 406:99-114.

Jones JF, Wang Y, Jordan D (1995) Heart rate responses to selective stimulation of cardiac vagal $\mathrm{C}$ fibres in anaesthetized cats, rats and rabbits. J Physiol (Lond) 489:203-214.

Jones JF, Wang Y, Jordan D (1998) Activity of C fibre cardiac vagal efferents in anaesthetized cats and rats. J Physiol (Lond) 507:869-880.

Kales A, Caldwell AB, Cadieux RJ, Vela-Bueno A, Ruch LG, Mayes SD (1985) Severe obstructive sleep apnea: II. Associated psychopathology and psychosocial consequences. J Chronic Dis 38:427-434.

Kawai Y, Qi J, Comer AM, Gibbons H, Win J, Lipski J (1999) Effects of cyanide and hypoxia on membrane currents in neurons acutely dissociated from the rostral ventrolateral medulla of the rat. Brain Res 830:246-257.

Kent BB, Drane JW, Bluminstein B, Manning JW (1972) A mathematical model to assess changes in the baroreceptor reflex. Cardiology 57:295-310

Kobayashi S, Conforti L, Zhu WH, Beitner-Johnson D, Millhorn DE (1999) Role of the D2 dopamine receptor in molecular adaptation to chronic hypoxia in PC12 cells. Pflügers Arch 438:750-759.

Loewy AD, Spyer KM (1990) Vagal preganglionic neurons. In: Central regulation of autonomic functions (Loewy AD, Spyer KM, eds), pp 68-87. New York: Oxford UP.

Machado BH (2001) Neurotransmission of the cardiovascular reflexes in the nucleus tractus solitarii of awake rats. Ann NY Acad Sci 940:179-196.

Magavi SS, Leavitt BR, Macklis JD (2000) Induction of neurogenesis in the neocortex of adult mice. Nature 405:951-955.

Nagata N, Saji M, Ito T, Ikeno S, Takahashi H, Terakawa N (2000) Repetitive intermittent hypoxia-ischemia and brain damage in neonatal rat. Brain Dev 22:315-320.

Nathan MA, Reis DJ (1977) Chronic labile hypertension produced by lesions of the nucleus tractus solitarii in the cat. Circ Res 40:72-81.

Nolan PC, Waldrop TG (1996) Ventrolateral medullary neurons show age-dependent depolarizations to hypoxia in vitro. Brain Res Dev Brain Res 91:111-120.

Ohtake PJ, Torres JE, Gozal YM, Graff GR, Gozal D (1998) NMDA receptors mediate cardiorespiratory responses to afferent peripheral chemoreceptor input in the conscious rat. J Appl Physiol 84:853-861.

Pappenheimer JR (1977) Sleep and respiration of rats during hypoxia. J Physiol (Lond) 266:191-207.

Rockhold RW, Caldwell RW (1979) Effect of lesions of the nucleus tractus solitarii on the cardiovascular actions of clonidine in conscious rats. Neuropharmacology 18:347-354. 
Roux J-C, Pequignot JM, Dumas S, Pascual O, Ghilini G, Pequignot J, Mallet J, Denavit-Saubie M (2000a) O2-sensing after carotid chemodenervation: hypoxic ventilatory responsiveness and upregulation of tyrosine hydroxylase mRNA in brainstem catecholaminergic cells. Eur J Neurosci 12:3181-3190.

Roux J-C, Pequignot J, Pascual O, Pequignot JM (2000b) Ventilatory and central neurochemical reorganization of $\mathrm{O} 2$ chemoreflex after carotid sinus nerve transection in rat. J Physiol (Lond) 522:493-501.

Sato MA, Menani JV, Lopes OU, Colombari E (1999) Commissural NTS lesions and cardiovascular responses in aortic baroreceptordenervated rats. Hypertension 34:739-743.

Schafer T, Morin-Surun MP, Denavit-Saubie M (1993) Oxygen supply and respiratory-like activity in the isolated perfused brainstem of the adult guinea pig. Brain Res 618:246-250.

Sun MK, Reis DJ (1994) Dopamine or transmitter release from rat carotid body may not be essential to hypoxic chemoreception. Am J Physiol 267:R1632-R163.
Sved AF (1986) Peripheral pressor systems in hypertension caused by nucleus tractus solitarius lesions. Hypertension 8:742-747.

Switzer III RC (2000) Application of silver degeneration stains for neurotoxicity testing. Toxicol Pathol 28:70-83.

Wang Y, Jones JF, Jeggo RD, de Burgh Daly M, Jordan D, Ramage AG (2000) Effect of pulmonary C-fibre afferent stimulation on cardiac vagal neurones in the nucleus ambiguus in anaesthetized cats. J Physiol (Lond) 526:157-165.

Weidner N, Ner A, Salimi N, Tuszynski MH (2001) Spontaneous corticospinal axonal plasticity and functional recovery after adult central nervous system injury. Proc Natl Acad Sci USA 98:3513-3518.

West MJ, Blessing WW, Chalmers J (1981) Arterial baroreceptor reflex function in the conscious rabbit after brainstem lesions coinciding with the A1 group of catecholamine neurons. Circ Res 49:959-970.

Zhang J, Mifflin SW (1998) Differential roles for NMDA and nonNMDA receptor subtypes in baroreceptor afferent integration in the nucleus of the solitary tract of the rat. J Physiol (Lond) 511:733-745. 